\title{
Editorial
}

\section{Intelligent Modeling and Verification}

\author{
Guiming Luo, ${ }^{1}$ Graziano Chesi, ${ }^{2}$ Xiaoyu Song, ${ }^{3}$ and Xiaojing Yang ${ }^{4}$ \\ ${ }^{1}$ School of Software, Tsinghua University, Beijing 100084, China \\ ${ }^{2}$ Department of Electrical and Electronic Engineering, University of Hong Kong, Pokfulam Road, Hong Kong \\ ${ }^{3}$ Maseeh College of Electrical and Computer Engineering, Portland State University, P.O. Box 751, Portland, OR 97207, USA \\ ${ }^{4}$ Department of Mathematical Sciences, Tsinghua University, Beijing 100084, China
}

Correspondence should be addressed to Guiming Luo; gluo@tsinghua.edu.cn

Received 27 August 2013; Accepted 27 August 2013

Copyright (C) 2013 Guiming Luo et al. This is an open access article distributed under the Creative Commons Attribution License, which permits unrestricted use, distribution, and reproduction in any medium, provided the original work is properly cited.

System modeling tends to have many complex features, and uncertainties often lead to numerous complications and influence many important aspects related to its applications. Intelligent modeling merges mathematical and computerbased approaches, and it utilizes pioneering new scientific methods and cutting-edge technologies.

In recent years, significant achievements have been made in system modeling and verification. This special issue concentrates on current developments in the areas of intelligent modeling, intelligent computing, and formal methods. After a high-quality peer-reviewing process, 27 papers have been selected and published in this special issue as follows.

The paper entitled "An algorithm for discretization of real value attributes based on interval similarity" (L. Zou et al.) defines an interval similarity function as a new merging standard in the process of discretization. The paper entitled "Recursive identification for dynamic linear systems from noisy Input-Output measurements" (D. Fan and K. Lo) deals with the adaptive identification problem of real-time EIV models. Based on the graph structure, the paper entitled "An effective heuristic based approach for partitioning” (X. Zhao et al.) constructs a communication graph for embedded system and describes the delay-related constraints and the cost-related objective. Between agents, the paper entitled "Proactive communicating process with asymmetry in multi-agent systems" (J. Wang et al.) presents a formalized communicating process to deal with information asymmetry. A Monte-Carlo reliability analysis method for MANET is presented in the paper entitled "A novel two-terminal reliability analysis for MANET" (X. Zhao et al.). Using a probabilistic analysis, the paper entitled "Reliable node clustering for mobile Ad Hoc networks"
(T. Wang and W. Hung) investigates clustering algorithm. Based on optical flow motion features extraction, the paper entitled "Action recognition by joint spatial-temporal motion feature" (W. Zhang et al.) introduces a method for human action recognition. Convergence and consistent properties of the EFOP method are shown in the paper entitled "An identification method based on EFOP and frequency domain smoothing" (Y. Zhang et al.). Using an enhanced mean shift method, an intelligent modeling method for perceptual spatial-space generation model is presented in the paper entitled "Spatial object tracking using an enhanced mean shift method based on perceptual spatial-space generation model" (P. Han et al.). The macroscopic approximation models are proposed in the paper entitled "Positive macroscopic approximation for fast attribute reduction" (Z. Lu et al.). All 3120218828 optimal 4-bit reversible circuits with up to 8 gates for the CNT are created in the paper entitled "Efficient algorithms for optimal 4-bit reversible logic system synthesis" (Z. Li et al.). A discrete-time, continuous-state Hopfield neural network with states being updated synchronously is presented in the paper entitled "Chaotic Hopfield neural network swarm optimization and its application" (Y. Sun et al.).

Furthermore, the paper entitled "Formalization of linear space theory in the higher-order logic proving system" (J. Zhang et al.) presents the formalization of the linear space theory in HOL4. The paper entitled "Bounded model checking of ETL cooperating with finite and looping automata connective" (R. Wang et al.) presents a semantic BMC encoding approach and an algorithm for the model checker ENuSMV. The paper entitled "Efficient semantics-based compliance checking using 
LTL formulae and unfolding" (L. Song et al.) formalizes some operational properties which contain the linearity, monotonicity, integration by parts, Cauchy-type integrability criterion and other important theorems of gauge integral in higher-order-logic 4 to verify an inverting integrator. The paper entitled "Verification of opacity and diagnosability for pushdown systems" (K. Kobayashi and K. Hiraishi) discusses verification of opacity and diagnosability for infinite-state DESs modeled by pushdown automata. The paper entitled "Component based formal modeling of PLC systems" (R. Wang et al.) proposes a systemic method to verify the construction of PLC model. An algorithm is presented in the paper entitled "A transformation-based approach to implication of GSTE assertion graphs" (G. Yang et al.) to transform a GSTE assertion graph to a finite-state automaton. Based on both Groebner bases approaches and symbolic simulation, the paper entitled "Algebraic verification method for SEREs properties via Groebner bases approaches" (N. Zhou et al.) presents an efficient solution to perform linear temporal properties verification for synchronous digital systems. A verification solution based on characteristic set of Wu's Method towards system Verilog assertion checking over digital circuit systems is proposed in the paper entitled "Wu's characteristic set method for system Verilog assertions verification" (X. Gao et al.). For static correctness checking of domain-specific errors, the paper entitled "OntCheck an ontology-driven static correctness checking tool for component-based models" (X. Lin et al.) proposes an ontology-driven tool. The MVB system modeling and verification are concerned in the paper entitled "Formal modeling and verification for MVB" (M. Xia et al.). The boom system is modeled and verified in the paper entitled "Automata-based analysis of stage suspended boom systems" (A. He et al.) by the hybrid automaton. The paper entitled "A unified framework for DPLL $(T)+$ certificates" (M. Zhou et al.) proposes a unified certificate framework based on DPLL(T). In software implementation, a latent implementation error detection approach is proposed in the paper entitled "The gauge integral theory in HOL4" (Z. Shi et al.) to detect latent errors. The paper entitled "A latent implementation error detection method for software validation" (J. Zhou et al.) defines a semantics-based process model query language through simplifying a property specification pattern system. A model reduction method based on the Colored Petri Net is shown in the paper entitled "A model reduction method for parallel software testing" (T. Sun and X. Ye).

\section{Acknowledgments}

We sincerely appreciate the participants in this special issue for their inspiring contributions, as well as the anonymous reviewers for their diligent work. In addition, we would like to express our appreciation to the editorial board members of this journal, for their help and support throughout the preparation of this special issue.

Guiming Luo

Graziano Chesi

Xiaoyu Song

Xiaojing Yang 


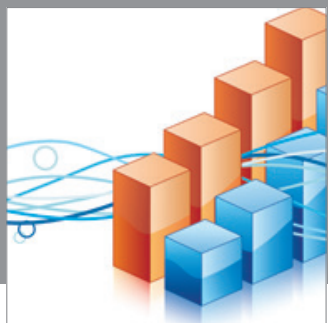

Advances in

Operations Research

mansans

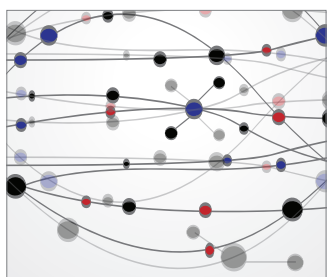

The Scientific World Journal
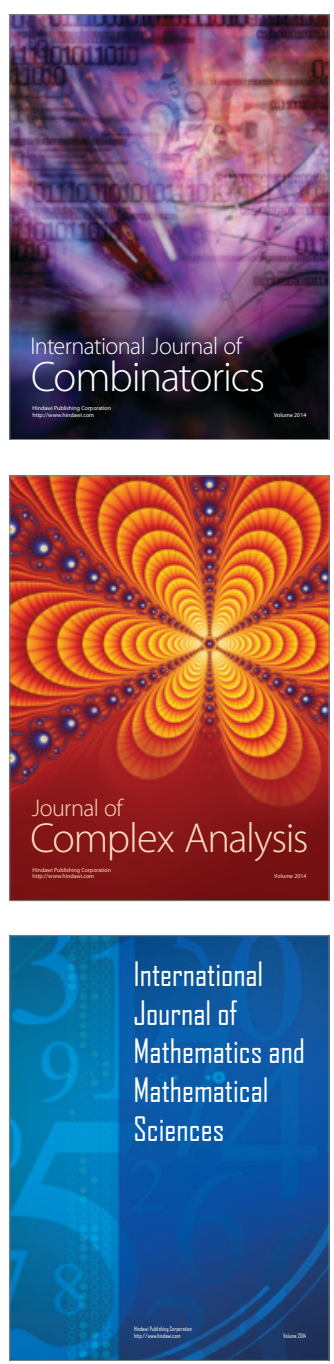
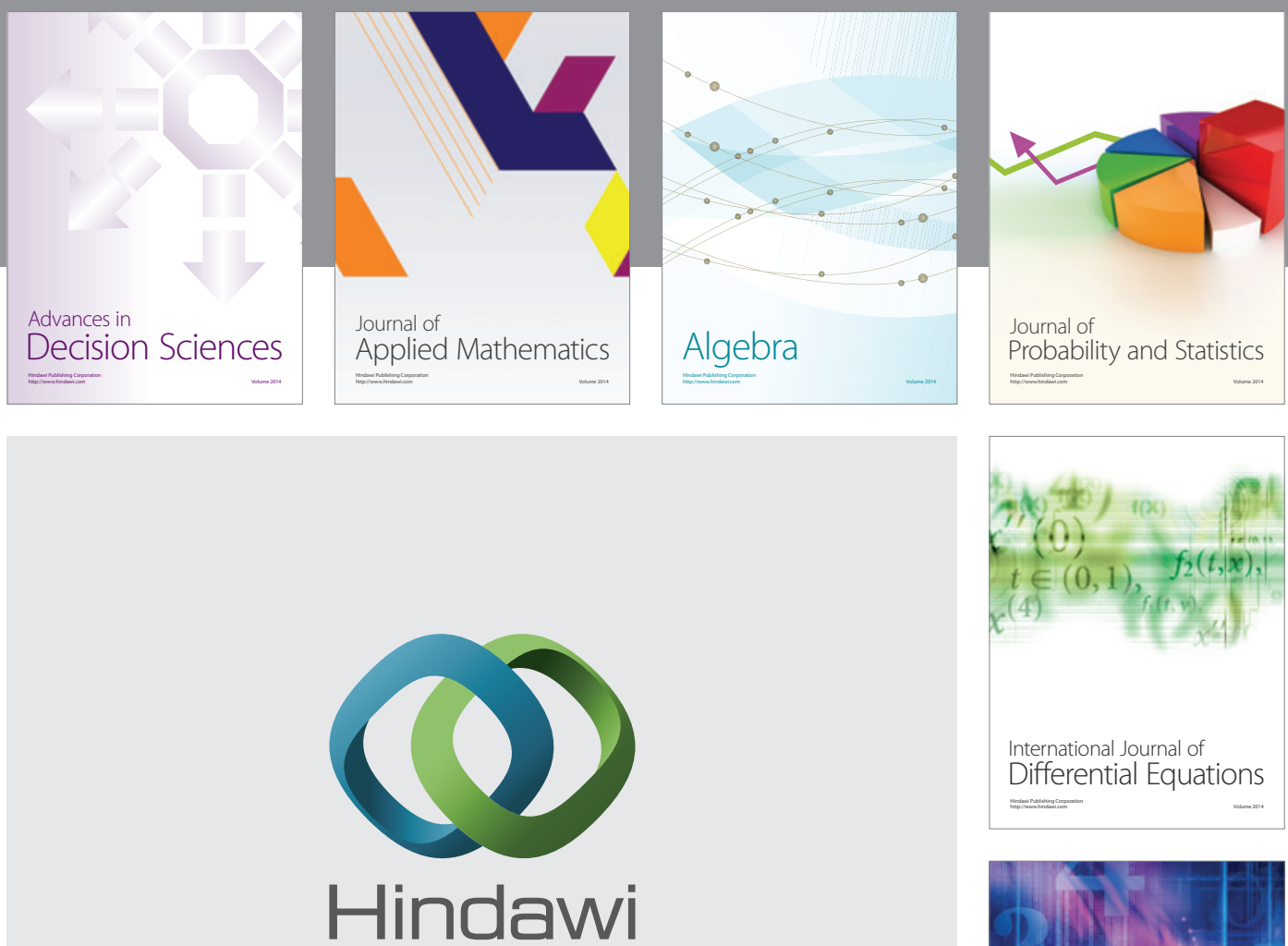

Submit your manuscripts at http://www.hindawi.com
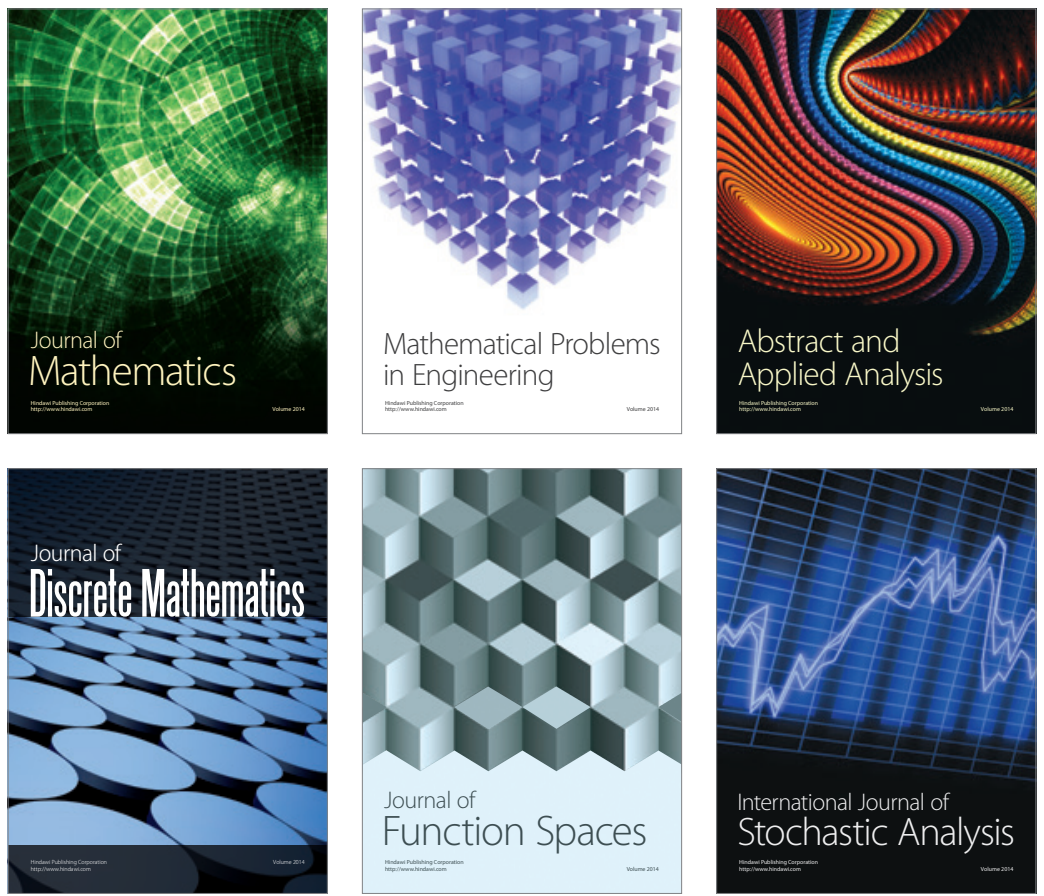

Journal of

Function Spaces

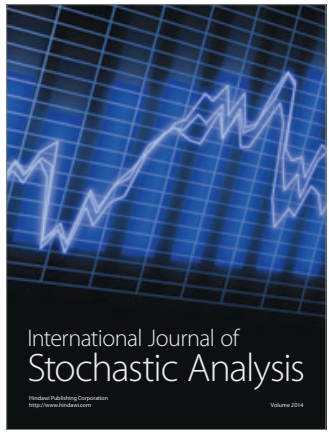

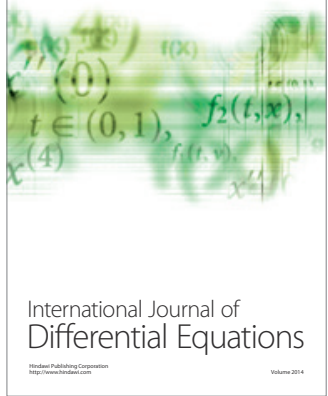
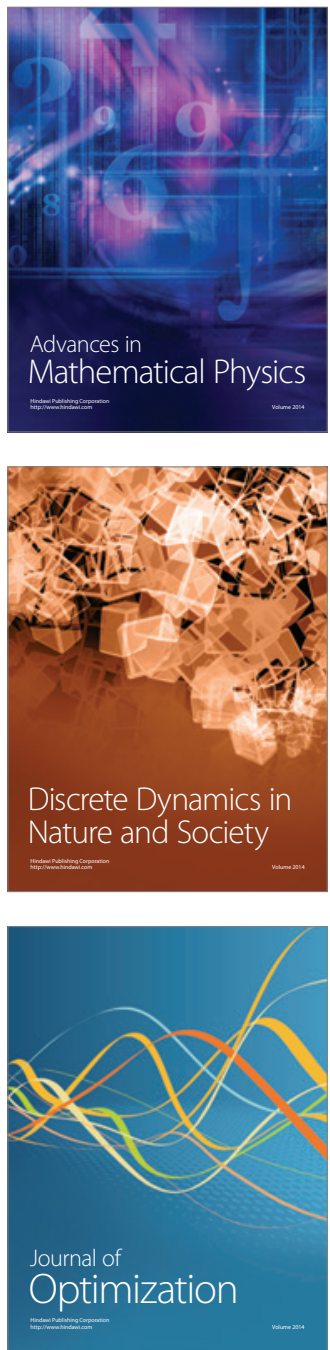